\title{
SÍNDROME DE BURNOUT EN PERSONAL DE ATENCIÓN A URGENCIAS MÉDICAS DURANTE LA PANDEMIA DE COVID-19
}

\author{
BURNOUT SYNDROME IN EMERGENCY CARE PERSONNEL DURING THE COVID-19 \\ PANDEMIC
}

\begin{abstract}
Janneth Aracely Valdivieso Maggi ${ }^{(1)}$ Darwin Raúl Noroña Salcedo ${ }^{(2)}$ Vladimir Vega Falcón ${ }^{(3)}$
(1) Hospital Provincial General Docente Riobamba, ${ }^{(2)}$ Universidad Regional Autónoma de los Andes, ${ }^{(3)}$ Universidad Regional Autónoma de los Andes
\end{abstract}

Email.: umsmwork74@gmail.com

DOI: https://doi.org/10.33789/talentos.8.1.146

\begin{abstract}
Resumen: El Sindrome de Burnout constituye un desorden funcional de la esfera neuropsiquiátrica condicionado por la exposición crónica a situaciones que general elevados niveles de estrés. La situación vivida por los trabajadores que brindaron asistencia médica de urgencia durante la pandemia de COVID-19 es consistente con un nivel de estrés elevado y mantenido. El objetivo de esta investigación fue identificar la presencia de Síndrome de Burnout en el personal de atención a urgencias médicas durante la pandemia de COVID-19. Para esto se realizó una investigación básica, descriptiva y explicativa que incluyó a 135 trabajadores de la salud que prestaron servicios en medicina de urgencia del Hospital provincial General Docente de Riobamba durante la pandemia de COVID-19. La muestra quedó conformada por 101 trabajadores a los cuales se les aplicó un cuestionario para características generales y la Escala de Maslach Burnout Inventory para identificar la presencia del Síndrome de Burnout. Existió un promedio de edad de 47,17 años, con predominio de trabajadoras (57,42\%) y de personal de enfermería (38,62\%). En el 35,64\% de los trabajadores se identificó la presencia de Síndrome de Burnout, siendo más frecuente en las féminas (77,78\%) y en el personal de enfermería (58,33\%). Se concluye que la situación vivida durante la pandemia de COVID-19
\end{abstract}

Recibido: 10 de enero de 2021

Aceptado: 20 de mayo de 2021

Publicado como artículo cientifico en la Revista de Investigación Talentos VIII (1), 93-100 
generó elevados niveles de estrés en el personal que brindó servicios de emergencia médica en el Hospital Provincial General Docente de Riobamba. Esta situación repercutió en el aumento de trabajadores de salud con diagnóstico de Síndrome de Burnout.

Palabras claves: COVID-19; Estrés laboral; Síndrome de Burnout.

Abstract: The Burnout Syndrome constitutes a functional disorder of the neuropsychiatric sphere conditioned by chronic exposure to situations that generate high levels of stress. The situation experienced by workers who provided emergency medical assistance during the COVID-19 pandemic is consistent with a high and sustained level of stress. The objective of this research was to identify the presence of Burnout Syndrome in emergency medical care personnel during the COVID-19 pandemic. For this, a basic, descriptive and explanatory research was conducted that included 135 health workers who provided emergency medicine services at the Riobamba Provincial General Teaching Hospital during the COVID-19 pandemic. The sample consisted of 101 workers to whom a questionnaire for general characteristics and the Maslach Burnout Inventory Scale were applied to identify the presence of Burnout Syndrome. There was an average age of 47.17 years, with a predominance of female workers $(57.42 \%)$ and nursing personnel (38.62\%). In 35.64\% of the workers the presence of Burnout Syndrome was identified, being more frequent in females (77.78\%) and in nursing personnel (58.33\%). It is concluded that the situation experienced during the COVID-19 pandemic generated high levels of stress in the personnel who provided medical emergency services at the Riobamba Provincial General Teaching Hospital. This situation had an impact on the increase in health workers diagnosed with Burnout Syndrome.

Key words:COVID-19; Work stress; Burnout syndrome.

\section{INTRODUCCIÓN}

A final del año 2019 el mundo se estremecía con la noticia de una nueva enfermedad identificada en la provincia china de Wuhan. La enfermedad, causada por un tipo específico de coronavirus fue bautizada como COVID-19 (Solis Cartas, 2020). La enfermedad rápidamente se propago a nivel mundial, los niveles de contagio fueron elevados y la enfermedad no solo comenzó a expandirse sino a condicionar gran cantidad de complicaciones y muertes (Chia Proenza, Gómez Conde, \& del Toro Ravelo, 2020). Estas características determinaron que en el mes de marzo la Organización Mundial de la Salud declaró a la COVID-19 como una pandemia. La enfermedad ha causado más de un millón de muertes hasta la actualidad y aún no existen esquemas terapéuticos capaces de prevenir las complicaciones de la enfermedad (Solis Cartas, \& Martinez Larrarte, 2020).

Desde el surgimiento de la enfermedad el personal de salud ha enfrentado innumerables retos; la incertidumbre relacionada con el esquema terapéutico a utilizar, el elevado número de contagios y muertes y la inexistencia de medidas de prevención fueron algunos de los retos diarios a los que se enfrentaron. Secundariamente comenzaron 
los contagios y muertes dentro del personal de salud dedicado a la atención de urgencia por COVID-19, lo que demandó del personal de salud un esfuerzo adicional para adaptarse a los tiempos de pandemia por COVID-19 (Pérez Abreu, Gómez Tejeda, \& Dieguez Guach, 2020).

Ecuador fue el segundo país del continente americano en reportar casos de COVID-19. La situación epidemiológica se hizo compleja rápidamente. La rápida propagación de la enfermedad, el elevado número de muertes y la creciente demanda de la población motivaron el colapso de los servicios de salud, públicos y privados, del país (Molina Prendes, \& Mejias Herrera, 2020).

El personal de salud dedicado a la atención de urgencia durante la pandemia de COVID-19 se enfrentó a situaciones muy difícil dentro de las que destacaron la muerte de familiares, amigos y compañeros de trabajo, intensas jornadas de trabajo, escases de recursos para el tratamiento, insuficientes número de camas hospitalarias y de equipos necesarios para la atención médica y otros. Todas estas situaciones generaron distintos niveles de estrés en el personal de salud; el cual se mantuvo durante los meses más críticos de la pandemia (Peraza de Aparicio, 2020).

Se reconoce que los niveles de estrés mantenido son capaces de producir una enfermedad conocida como Síndrome de Burnout. Este término fue introducido en el año en 1974 por Herbert Freudenberger, y se describe como un trastorno que aparece secundario al estrés laboral crónico. Sus principales caráctersiticas clínicas incluyen el agotamiento emocional, síntomas físicos, actitud cínica o distante frente al trabajo (despersonalización), sensación de ineficacia y pérdida de habilidades para la comunicación. El Síndrome de Burnout se incluye entre los principales problemas de salud mental y es considerado por muchos investigadores como la antesala de varias afecciones psiquiátricas motivadas por un estrés mantenido (Rodríguez Ramírez, Guevara Araiza, \& Viramontes Anaya, 2017).

Es por esto que teniendo en cuenta la situación vivida por el personal de salud dedicado a la atención de urgencia durante la pandemia de COVID-19, el estrés mantenido al que estuvieron sometidos durante varios meses y la posibilidad de que muchos de ellos estén expresando manifestaciones clínicas compatible con síndrome de Burnout; se decide realizar esta investigación con el objetivo de identificar la presencia de Síndrome de Burnout en el personal de atención a urgencias médicas durante la pandemia de COVID-19.

\section{METODOLOGÍA}

Se realizó una investigación básica, no experimental, de corte transversal y con un diseño descriptivo y explicativo. El enfoque de la investigación fue mixto ya que incluyó elementos cualitativos y cuantitativos. E1 universo estuvo constituido por un total de 135 trabajadores de la salud dedicados al servicio de urgencia durante los meses de confinamiento por la pandemia de COVID-19 en unidades de salud de la ciudad de Riobamba. Después de realizar los cálculos matemáticos se definió la muestra en un total 
de 101 trabajadores cumplieron los criterios de inclusión y exclusión definidos para el estudio

\section{Criterios de inclusión}

Trabajadores que laboraron en el servicio de urgencia durante los meses de confinamiento por la COVID-19 en el Hospital Provincial general Docente de Riobamba.

Personas que estuvieron de acuerdo en participar en la investigación y lo expresaron mediante la firma del consentimiento informado.

Criterios de exclusión

Personas que no estuvieron de acuerdo en participar en la investigación por lo que no procedieron con la firma del consentimiento informado.

Para conformar la muestra se utilizó el método de muestreo aleatorio simple garantizando que cada trabajador tuviera las mismas oportunidades para formar parte de la muestra de la investigación.

Para la realización de la investigación se tuvieron en cuenta las siguientes variables de investigación: características generales y presencia de Síndrome de Burnout. La variable caráctersiticas generales presentó las siguientes subvariables: edad, sexo y ocupación.

Como técnicas de investigación fueron utilizadas la revisión documental y la entrevista. La revisión documental facilitó el estudio de los elementos generales y particulares relacionados con el síndrome de Burnout. La entrevista se realizó como acompañamiento de la aplicación del instrumento de investigación, así como para aclarar términos y dudas que pudieran tener los trabajadores relacionadas con los objetivos y métodos de la investigación. Se realizaron entrevistas individuales y grupales, cumpliendo distintos fines en cada tipo de entrevista realizada.

El instrumento de investigación utilizado fue la Escala de Maslach Burnout Inventory. Esta escala se encuentra validad en idioma español e inglés y cuanta con un total de 22 preguntas con respuesta tipo Likert orientadas hacia 3 dominios principales: emocional, despersonalización y rendimiento laboral.

El procesamiento de la información se realizó de forma automatizada con la ayuda del programa estadísticos SPSS en su versión 20,5 para Windows. Se determinaron medidas de tendencia central y de dispersión para las variables cuantitativas y frecuencias absolutas y porcentajes para las variables cualitativas. Se definió el nivel de confianza en el 95\%, el margen de error en el 5\% y la significación estadística en una $\mathrm{p} \leq 0,05$. Todos los resultados fueron expresados en forma de tablas estadísticas para facilitar su comprensión.

Durante el desarrollo de la investigación se tuvieron en cuenta las normas y procedimientos establecidos en la declaración de Helsinki II para la realización de investigaciones en seres humanos. Los datos obtenidos fueron utilizados únicamente con fines investigativos y no se utilizaron datos de 
identificación personal durante el desarrollo de la investigación. La incorporación de los trabajadores fue totalmente voluntaria y no representó gasto alguno para ellos; su incorporación se produjo posterior a la firma del consentimiento informado. La base de datos, con toda la información recopilada fue destruida al terminar el informe final de la investigación.

\section{RESULTADOS Y DISCUSIÓN}

Tabla 1. Distribución de trabajadores según características generales.

\begin{tabular}{lc}
\hline \multicolumn{1}{c}{ Características } & $\begin{array}{c}\text { Muestra total de 101 } \\
\text { trabajadores } \\
\text { Frecuencia (por } \\
\text { ciento) }\end{array}$ \\
\hline Promedio de edad (años) & $\begin{array}{c}47,17 \text { años *DE } \\
18,83\end{array}$ \\
\hline \multicolumn{1}{c}{ Grupo de edades } \\
\hline Entre 20 y 39 años & $26(25,74)$ \\
Entre 40 y 59 años & $62(61,39)$ \\
De 60 años y más & $13(12,87)$ \\
\hline \multicolumn{2}{c}{ Sexo } \\
\hline Masculino & $43(42,58)$ \\
Femenino & $58(57,42)$ \\
\hline \multicolumn{2}{c}{ Ocupación } \\
\hline Personal médico & $22(21,78)$ \\
Personal de enfermería & $39(38,62)$ \\
Personal de servicio & $27(26,73)$ \\
Personal administrativo & $13(12,87)$ \\
\hline Fuente: cuestionario de investigación & $* D E:$ desviación \\
estándar &
\end{tabular}

El análisis de las características generales de los trabajadores de salud que participaron en el estudio (tabla 1) mostró un promedio de edad de 47,17 años con una DE de 18,83 años. Existió predominio de trabajadores entre 40 y 59 años (61,39\%) seguido del grupo de 20 a 39 años con un $25,74 \%$ del total de trabajadores investigados. En relación al sexo predominaron las féminas $(57,42 \%)$ en relación al sexo masculino $(42,58 \%)$. El $38,62 \%$ de las personas encuestadas correspondían al personal de enfermería, el $26,73 \%$ se desempeñaba como personal de servicio y el $21,78 \%$ como personal médico. Solo el $12,87 \%$ de los trabajadores que participaron en el estudio correspondieron a la categoría de personal administrativo.

Tabla 2. Distribución de trabajadores según presencia o ausencia de Síndrome de Burnout

\begin{tabular}{lcc}
\hline Síndrome & de & \multicolumn{2}{c}{$\mathbf{n = 1 0 1}$ trabajadores } \\
\cline { 2 - 3 } Burnout & Frecuencia & Por ciento \\
\hline Presencia & 36 & 35,64 \\
Ausencia & 65 & 64,36 \\
\hline Total & 101 & 100 \\
\hline
\end{tabular}

Fuente: Cuestionario de investigación

Al analizar la presencia de Síndrome de Burnout en los trabajadores se obtuvo que en el $35,64 \%$ se confirmó la presencia del síndrome; en el restante $64,36 \%$ de los trabajadores no se identificó la presencia del síndrome.

Tabla 3. Distribución de trabajadores según presencia de Síndrome de Burnout y características generales.

\begin{tabular}{|c|c|c|c|}
\hline \multirow[t]{2}{*}{$\begin{array}{l}\text { Característi- } \\
\text { cas generales }\end{array}$} & \multicolumn{3}{|c|}{$\begin{array}{c}\text { Total de trabajadores con Sín- } \\
\text { drome de Burnout } n=36\end{array}$} \\
\hline & Frecuencia & Por ciento & $\mathbf{p}$ \\
\hline $\begin{array}{l}\text { Grupo de } \\
\text { edades }\end{array}$ & & $n=36$ & \\
\hline $\begin{array}{l}\text { Entre } 20 \text { y } 39 \\
\text { años }\end{array}$ & 4 & 11,11 & 0,09 \\
\hline $\begin{array}{l}\text { Entre } 40 \text { y } 59 \\
\text { años }\end{array}$ & 30 & 83,33 & 0,017 \\
\hline $\begin{array}{l}\text { De } 60 \text { años y } \\
\text { más }\end{array}$ & 2 & 5,56 & 0,095 \\
\hline Sexo & & $n=36$ & \\
\hline Masculino & 8 & 22,22 & ---- \\
\hline Femenino & 28 & 77,78 & ---- \\
\hline Ocupación & & $n=36$ & \\
\hline $\begin{array}{l}\text { Personal } \\
\text { médico }\end{array}$ & 9 & 25,00 & 0,075 \\
\hline
\end{tabular}




\begin{tabular}{lccc}
$\begin{array}{l}\text { Personal de } \\
\text { enfermería }\end{array}$ & 21 & 58,33 & 0,041 \\
$\begin{array}{l}\text { Personal de } \\
\text { servicio }\end{array}$ & 5 & 13,89 & 0,086 \\
$\begin{array}{l}\text { Personal ad- } \\
\text { ministrativo }\end{array}$ & 1 & 2,78 & 0,101 \\
\hline $\begin{array}{l}\text { Fuente: } \text { ELCSA } \\
{ }^{*} p \leq 0,05\end{array}$ & & &
\end{tabular}

Al realizar el análisis bivariado incluyendo la presencia de Síndrome de Burnout y las características generales se puede observar en la tabla 3 que en relación a la edad el $83,33 \%$ de los trabajadores en los que se identificó Síndrome de Burnout tenían entre 40 y 59 años de edad. El 77,78\% son del sexo femenino y el 58,33\% corresponden a personal de enfermería, mientras que el $25,0 \%$ de las personas identificadas como positivas al síndrome eran médicos.

\section{DISCUSIÓN}

El Síndrome de Burnout constituye un conjunto de manifestaciones clínicas resultantes de un nivel de estrés mantenido; se considera como factor desencadenante de otros trastornos neuropsiquiátricos como el síndrome depresivo, la bipolaridad, conducta maniaco depresiva y trastornos disociativos entre otras (Malander, 2016). Es por eso que se considera de gran importancia la determinación del síndrome en estadios tempranos y más aún de las principales condiciones que generan el estrés mantenido y de esa forma contribuir al adecuado estado de salud biopsicosocial de la población.

El análisis de las características generales de los trabajadores mostró un promedio de edad ligeramente superior a los 45 años; este resultado concuerda con el perfil laboral de las principales actividades a desarrollar dentro de la prestación de servicios médicos de urgencias. El predominio de trabajadoras también es un resultado esperado y visto en la práctica en la mayoría de las instituciones de salud. En cuanto a la categoría ocupacional destacó el predominio del personal de enfermería y de servicios, seguido por el personal médico y en menor medida el personal administrativo.

Este resultado está condicionado por la propia actividad que se realiza en las unidades médicas emergentes, donde el personal de mayor presencia corresponde a personal de enfermería y servicios. Estos datos son difíciles de comparar con otros estudios pero, según la experiencia previa del equipo de investigación corresponde con la situación de otras entidades de salud, públicas y privadas.

El análisis de la presencia de Síndrome de Burnout constituyó el objetivo fundamental de la investigación, el porcentaje encontrado de presencia de la enfermedad es considerado como elevado, teniendo en cuenta referentes internacionales que muestran una presencia del síndrome entre el 10 y $15 \%$ de los trabajadores de la salud (Solís Chuquiyauri, Zamudio Eslava, Matzumura Kasano, \& Gutiérrez Crespo, 2016). Es importante destacar que las cifras de comparación que se mencionan corresponden a estudios previos a la pandemia de COVID-19.

El elevado por ciento de presencia de la enfermedad identificado guarda relación con la difícil situación epidemiológica y social que se vivió durante los meses de marzo a julio del año 2020. Los trabajadores de la salud encargados de la atención de 
urgencia tuvieron que afrontar situaciones desagradables que incluyeron el aumento de la demanda de servicios, extensión de las jornadas laborales, la incertidumbre sobre los esquemas farmacológicos a utilizar, la escasez de recursos terapéuticos, instrumentos, equipamiento, camas hospitalarias $\mathrm{y}$ medicamentos; así como el contagio y muerte de conocidos, amigos, compañeros de trabajo $\mathrm{y}$ familiares.

Este tipo de situaciones aún se mantiene, pero en menor escala que en meses anteriores; por lo que la situación estresante fue mantenida al menos durante 5 meses, tiempo suficiente para comprometer los mecanismos adaptativos de muchas personas. De esta forma es como la presencia de un estrés mantenido genera disfunción adaptativa y aparición de manifestaciones clínicas compatibles con la enfermedad.

Existen estudios que abordan el tema de la presencia de Síndrome de Burnout y sus manifestaciones clínicas. De esta forma autores como Murat y Solmaz reportan elevado por ciento de presencia de manifestaciones compatibles con la enfermedad en población adulta. También autores como Jalili y colaboradores (2020) identifican más del 50\% de trabajadores de la salud con presencia del síndrome.

En relación al análisis relazado entre características generales y presencia de la enfermedad se obtuvo predominio en trabajadoras, entre 40 y 59 años de edad y enfermeras. Aunque no existen estudios que reporten resultados similares que posibiliten hacer comparaciones es importante destacar que el personal de enfermería es vital en el correcto funcionamiento de los servicios de emergencia, más aún en etapa de pandemia. Durante este periodo de tiempo el personal de enfermería estuvo en la primera línea de enfrentamiento a los pacientes afectos por COVID-19, por lo que sufrieron directamente las dificultades y carencias para poder realizar su trabajo, lo que puede haber significado el detonante para el colapso de los mecanismos adaptativos y la aparición de las manifestaciones clínicas.

\section{CONCLUSIÓN}

Se concluye que la situación vivida durante la pandemia de COVID-19 generó elevados niveles de estrés en el personal que brindó servicios de emergencia médica en el Hospital Provincial General Docente de Riobamba. Esta situación repercutió en el aumento de trabajadores de salud con diagnóstico de Síndrome de Burnout.

\section{REFERENCIAS BIBLIOGRÁFICAS}

Chia Proenza, D., Gómez Conde, S.Y., \& del Toro Ravelo, L.M. (2020). Acercamiento a la COVID-19 desde una perspectiva pediátrica. Revista Cubana de Reumatología, 22(2), e830. Epub 01 de agosto de 2020. Recuperado de http://scielo.sld.cu/scielo. php? script $=$ sci_arttext\&pid $=\mathrm{S} 1817$ $\underline{59962020000200015 \& \operatorname{lng}=\mathrm{es} \& \mathrm{t} \operatorname{lng}=\mathrm{pt}}$

Malander, N.M. (2016). Síndrome de Burnout y Satisfacción Laboral en Docentes de Nivel Secundario. Ciencia \& trabajo, 18(57), 177-182. Recuperado de $\quad$ https://dx.doi.org/10.4067/ S $0718-24492016000300177$

Molina Prendes, N., \& Mejias Herrera, M.L. (2020). Impacto social de la 
COVID-19 en Brasil y Ecuador: donde la realidad supera las estadísticas. EDUMECENTRO, 12(3), 277-283. Epub 22 de junio de 2020. Recuperado de http://scielo.sld.cu/scielo. php? script $=$ sci arttext\&pid $=$ S207728742020000300277\&lng=es\&tlng $=$ pt

Murat, Y., \& Solmaz, F. (2020). COVID-19 burnout, COVID-19 stress and resilience: Initial psychometric properties of COVID-19 Burnout Scale. Death Studies. Publicado en línea. Recuperado de: https:// www.tandfonline.com/doi/full/1 $\underline{0.1080 / 07481187.2020 .1818885}$

Peraza de Aparicio, C.X. (2020). Salud laboral frente a la pandemia del COVID-19 en Ecuador. MediSur, 18(3), 507-511. Epub 02 de junio de 2020. Recuperado en 13 de diciembre de 2020, de http:// scielo.sld.cu/scielo.php?script $=$ sci arttt\&pid=S 1727-897X2020 $\underline{000300507 \& \operatorname{lng}=\mathrm{es} \& \mathrm{t} \operatorname{lng}=\mathrm{pt}}$

Pérez Abreu, M.R., Gómez Tejeda, J.J., \& Dieguez Guach, R.A. (2020). Características clínico-epidemiológicas de la COVID-19. Revista Habanera de Ciencias Médicas, 19(2), e3254. Epub 22 de abril de 2020. Recuperado de http:// scielo.sld.cu/scielo.php?script $=$ sci arttext\&pid=S 1729-519X202 $0000200005 \& \operatorname{lng}=\mathrm{es} \& \mathrm{t} \operatorname{lng}=\mathrm{es}$

Rodríguez Ramírez, J.A., Guevara Araiza, A., \& Viramontes Anaya, E. (2017). Síndrome de burnout en docentes. IE Revista de investigación educativa de la REDIECH, 8(14), 45-67. Recuperado de http://www.scielo.org.mx/scielo. php? script $=$ sci arttext\&pid $=S 2448$ $\underline{8502017000100045 \& \operatorname{lng}=\text { es\&tlng }=\mathrm{es}}$

Solis Cartas, U., \& Martinez Larrarte, J.P. (2020). Therapeutic options to cytokine release syndrome in patients with COVID-19. Revista Cubana de Medicina Militar, 49(3), e783. Epub 25 de noviembre de 2020. Recuperado de http://scielo.sld.cu/scielo. php? script $=$ sci arttext\&pid $=S 0138$ $65572020000300022 \& 1 \mathrm{n}$ $\mathrm{g}=\mathrm{e} \quad \mathrm{s} \& \mathrm{\&} \quad \mathrm{l} n \mathrm{~g}=\mathrm{e} \mathrm{n}$

Solis Cartas, U. (2020). Coronavirus y enfermedades reumáticas, suposiciones, mitos y realidades. Revista Cubana de Reumatología, 22(2), e791. Epub 01 de agosto de 2020. Recuperado en 13 de diciembre de 2020, de http://scielo.sld.cu/scielo. php? script $=$ sci arttext\&pid $=\mathrm{S} 1817$ 59962020000200001\&lng=es\&tlng $=\mathrm{es}$

Solís Chuquiyauri, Z., Zamudio Eslava, L., Matzumura Kasano, J., \& Gutiérrez Crespo, H. (2016). Relación entre clima organizacional y síndrome de burnout en el servicio de emergencia de un hospital Categoría III-2. Lima, Perú 2015. Horizonte Médico (Lima), 16(4), 32-38. Recuperado en 13 de diciembre de 2020, de http://www. scielo.org.pe/scielo.php? script $=$ sci arttext\&pid $=$ S $1727-558$ X201 $6000400006 \& \operatorname{lng}=$ es \& t lng $=$ es 